\title{
Determining Predictive Factors of Para-aortic Lymph Node Involvement in Low-grade Endometrial Cancer
}

\author{
Devon Smith ${ }^{1 *}$, Isabella Silvestre ${ }^{2}$, Elena Safronova ${ }^{1}$, Julieta Barroeta ${ }^{3}$, David Warshal ${ }^{4}$, Meredith Duffy $^{4}$, \\ Lauren Krill ${ }^{4}$, Robin Wilson-Smith ${ }^{5}$ and James Aikins ${ }^{4}$
}

${ }^{1}$ Department of Obstetrics and Gynecology, Cooper University Hospital, USA

${ }^{2}$ Cooper Medical School, Rowan University, USA

${ }^{3}$ Department of Pathology, Cooper University Hospital, USA

${ }^{4}$ Department of Gynecology Oncology, Cooper University Hospital, USA

${ }^{5}$ Department of Gynecology Oncology, Jefferson Health New Jersey, USA

*Corresponding author: Devon Smith, Department of Obstetrics and Gynecology,

Cooper University Hospital, Cooper Plaza, Suite 221, Camden, NJ 08103, USA.

Received Date: September 07, 2020

Published Date: September 22, 2020

\begin{abstract}
Objectives: The purpose of this study was to determine predictive factors of para-aortic lymph node (PALN) involvement in low-grade endometrial cancer of endometrioid type. These factors may influence treatment algorithms and determine the extent of lymph node dissection in patients with low grade endometrial cancer. Our hypothesis was that tumor size, location, and depth of myometrium invasion may be independent predictors of PALN involvement in low grade endometrial cancer.

Methods: A retrospective chart review was performed on patients with grades 1 or 2 endometrial cancer of endometroid type who underwent hysterectomy with lymph node removal from January 1, 2004 to August 1, 2014. Data was evaluated using independent t-tests, Mann Whitney U test, and chi square tests. Sensitivity, specificity, positive and negative predictive values were calculated for tumor size, location, and myometrium invasion in association with PALN and pelvic lymph node (PLN) involvement.

Results: A total of 259 patients met the inclusion criteria. Tumor size was not significantly different between positive and negative PALN samples ( $4.5 \mathrm{~cm}$ vs $3.5 \mathrm{~cm}$, respectively; $\mathrm{p}=0.29$ ). Location was not significantly different among positive and negative PALN groups, as the majority of patients in both groups had tumors in the fundal region ( $75 \%$ vs $70.5 \%$, respectively; $\mathrm{p}=1.00)$. Myometrial invasion was not significantly different between positive and negative PALN groups ( $48 \%$ vs $28 \%$, respectively; $\mathrm{p}=0.14$ ). Myometrial invasion was significantly different among positive and negative PLN groups $(71 \%$ vs $26 \%$, respectively. $\mathrm{p}<0.001)$. Lymphovascular space invasion (LVSI) demonstrated a significant association with both positive PALN and PLN groups ( $100 \%$ vs $22.31 \%$, and $81.25 \%$ vs $19.67 \%$, respectively; $\mathrm{p}=0.003,<0.001)$.
\end{abstract}

Conclusions: LVSI may be an independent predictor of both PALN and PLN involvement in low grade endometrial tumors. It remains unclear whether tumor size, location, and myometrial invasion can be used to predict para-aortic nodal involvement in these cases.

Keywords: Low grade endometrial cancer; Endometrioid adenocarcinoma; Para-aortic lymph nodes; Nodal metastasis; Tumor size; Tumor location; Myometrial invasion; Lymphovascular space invasion

\section{Introduction}

Endometrial cancer is the 4th leading cause of cancer among women and the most common malignancy among the female reproductive organs in the United States [1,2]. It is essential to

determine the most effective way to both correctly diagnose and subsequently treat affected patients. Treatment is founded on proper staging, and the extent of lymph node involvement is a 
critical determinant of tumor stage. Prior authors have attempted to identify predictive factors of pelvic lymph node (PLN) involvement in low grade endometrial cancer [3-7]. However, there is a paucity of research regarding factors such as tumor location, associated specifically with para-aortic lymph node (PALN) involvement in low grade tumors. This is likely due to variations in incidence rates. The overall frequency of PALN dissemination within endometrial adenocarcinoma has been reported as low and also as high as $17 \%$, depending on the tumor stage [4,7-10]. Further delineation of these factors may potentially help guide surgeons in the extent of required surgical dissection. The primary purpose of this study is to evaluate the following factors and their correlation to PALN involvement in low grade endometrial adenocarcinoma of endometrioid type: myometrial invasion, tumor size, tumor location, and lymphovascular space invasion (LVSI). By characterizing these variables, we can further risk stratify patients who may require additional surgical dissection and extensive nodal biopsy.

\section{Materials and Methods}

This study was a retrospective chart review designed to assess the best predictive factors of PALN involvement in low grade endometrial cancer. The protocol and all study materials were submitted and approved by the university's Institutional Review Board. Approval was obtained before any subjects were enrolled. The study was conducted in accordance with the protocol, applicable regulations, and guidelines governing clinical study conduct. To protect subjects' confidentiality, all subjects were assigned a numerical study identifier.

A chart review was performed for all women admitted to Cooper University Hospital with endometrial cancer and subsequent hysterectomy in the identified time frame. Included patients demonstrated grades 1 or 2 endometrial adenocarcinoma with primary histology of endometroid type with surgical lymph node removal during hysterectomy, nonspecific to operative approach, from January 1, 2004 to August 1, 2014. Operative approaches included open total abdominal hysterectomy, robotic hysterectomy, and laparoscopic assisted vaginal hysterectomy. Patients with primary histology of endometrioid cancer and secondary histology of alternative endometrial pathology were still included. Charts were abstracted for demographics such as age, which helped to define the patient population. Exclusion criteria included patients with concurrent gynecologic malignancies such as cervical or ovarian, any non-endometrioid primary histology, and any extensively incomplete pathology or operative reports. No criteria for patient follow up was included as this was strictly a retrospective chart review.

Demographic data and presurgical tumor grade were extracted from individual charts. Size of the tumor, location of the tumor, histology, PALN involvement, PLN involvement, the number of nodes removed, lymphovascular space involvement (LVSI), tumor stage, and the depth of myometrial invasion were all collected directly from pathology reports.
Tumor size was defined as the largest dimension of the tumor listed in the pathology report, in centimeters $(\mathrm{cm})$. If the tumor was listed as "unmeasurable" due to small size, it was reported as $0.1 \mathrm{~cm}$ for statistical purposes. Final statistics categorized tumor size as greater or less than $5 \mathrm{~cm}$. Tumor location was categorized as "fundus" or "lower uterine segment". By standards directly defined by the university board-certified pathologist involved in the study, if the pathology report did not clearly define either location, the measurement of $<3.5 \mathrm{~cm}$ from ectocervix was used as a cutoff for lower uterine segment location. If no exact measurement was listed but was reported as "close to endocervical junction" or "involved in the lower uterine segment," the specimen was categorized as lower uterine segment. Depth of myometrial invasion was reported as a percentage, either directly stated in the pathology report or calculated from the total myometrial depth.

Data was then evaluated using independent t-tests to compare continuous variables with normal distribution, Mann Whitney U to compare non-parametric continuous variables, and chi square tests to compare proportions. We also evaluated positive and negative predictive values, sensitivity and specificity to examine the predictive values of tumor size, tumor location and myometrium invasion in para-aortic lymph node and pelvic node involvement in the selected cases.

\section{Results}

In total, 498 patient charts were reviewed over the given time period. Of these charts, primary histology was reviewed providing the following findings; 418 endometrioid adenocarcinoma, 63 papillary serous carcinoma, 13 malignant mullerian mixed tumor, 2 clear cell carcinomas, and 2 specimens classified as mixed primary histology (papillary serous with clear cell and mixed papillary serous with endometrioid). Of the patients with primary histology of endometrioid adenocarcinoma, 367 were listed as grade 1 or 2 tumors, and 51 were grade 3. Pelvic and/or para-aortic lymph node sampling was performed on 269 specimens of the grade 1 and 2 tumors. Ten charts were then excluded due to concurrent ovarian or cervical cancer or an extensive amount of missing data from the chart or pathology report. These patients then met inclusion criteria and were included in this retrospective chart review. There were 162 Grade 1 tumors and 97 Grade 2 tumors. Four patients were identified with positive PALN involvement, 16 patients were identified with positive PLN involvement, and 1 patient had both positive PALN and PLN identified. All positive samples were reported by Cooper University Hospital's Department of Pathology (Figure 1).

\section{Tumor size}

Primary outcome compared tumor size with PALN involvement, with additional findings comparing tumor size and PLN involvement. Tumor size was not found to be statistically significantly between positive and negative PALN samples. Mean tumor size for paraaortic positive tumors was $4.12 \mathrm{~cm}(+/-2.0)$ and the median was 
$4.5 \mathrm{~cm}$. The mean tumor size for para-aortic negative tumors was $3.9 \mathrm{~cm} \mathrm{(+/-3.7)} \mathrm{with} \mathrm{a} \mathrm{median} \mathrm{of} 3.5 \mathrm{~cm} \mathrm{(p=0.29,} \mathrm{Figure} \mathrm{2).}$ Tumors measuring $5 \mathrm{~cm}$ or more were found to have the following associations with positive PALN involvement: a positive predictive value (PPV) of $2.94 \%$, a negative predictive value (NPV) of $98.92 \%$, sensitivity of $50.00 \%$ and specificity of $73.60 \%$ (Figure 3 ). Tumors of $5 \mathrm{~cm}$ or more were found to have the following associations with positive PLN involvement: PPV of $14.71 \%$, NPV of $96.77 \%$, sensitivity of $62.50 \%$ and specificity of $75.63 \%$ (Figure 4 ).

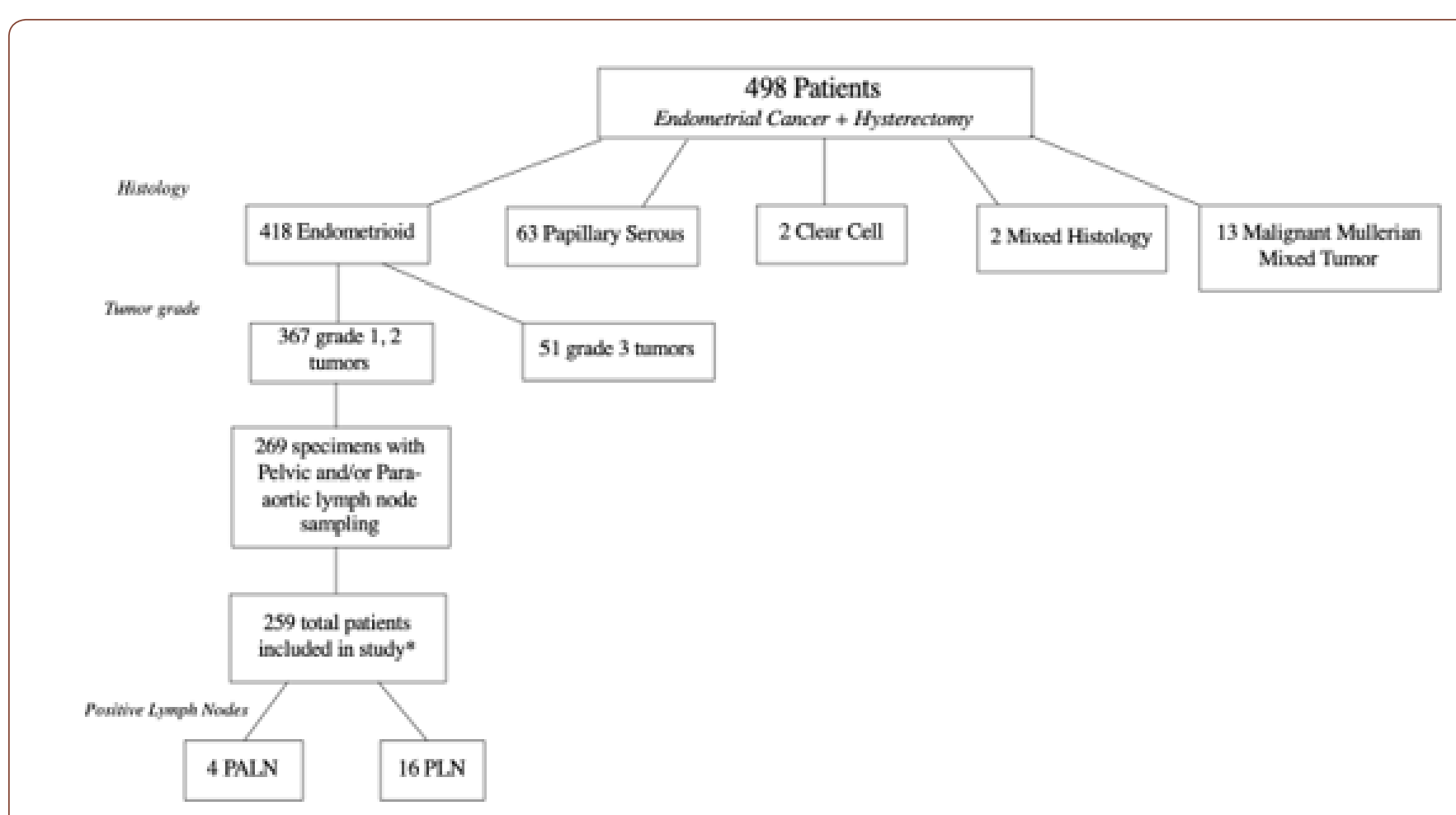

Figure 1: Inclusion and exclusion criteria for patient selection.

*259 patients included in study; Grade 1, 2 endometrioid adenocarcinoma with pelvic and/or para-aortic lymph nodes sampled. 10 charts excluded due to extensive missing data or concurrent additional cancer.

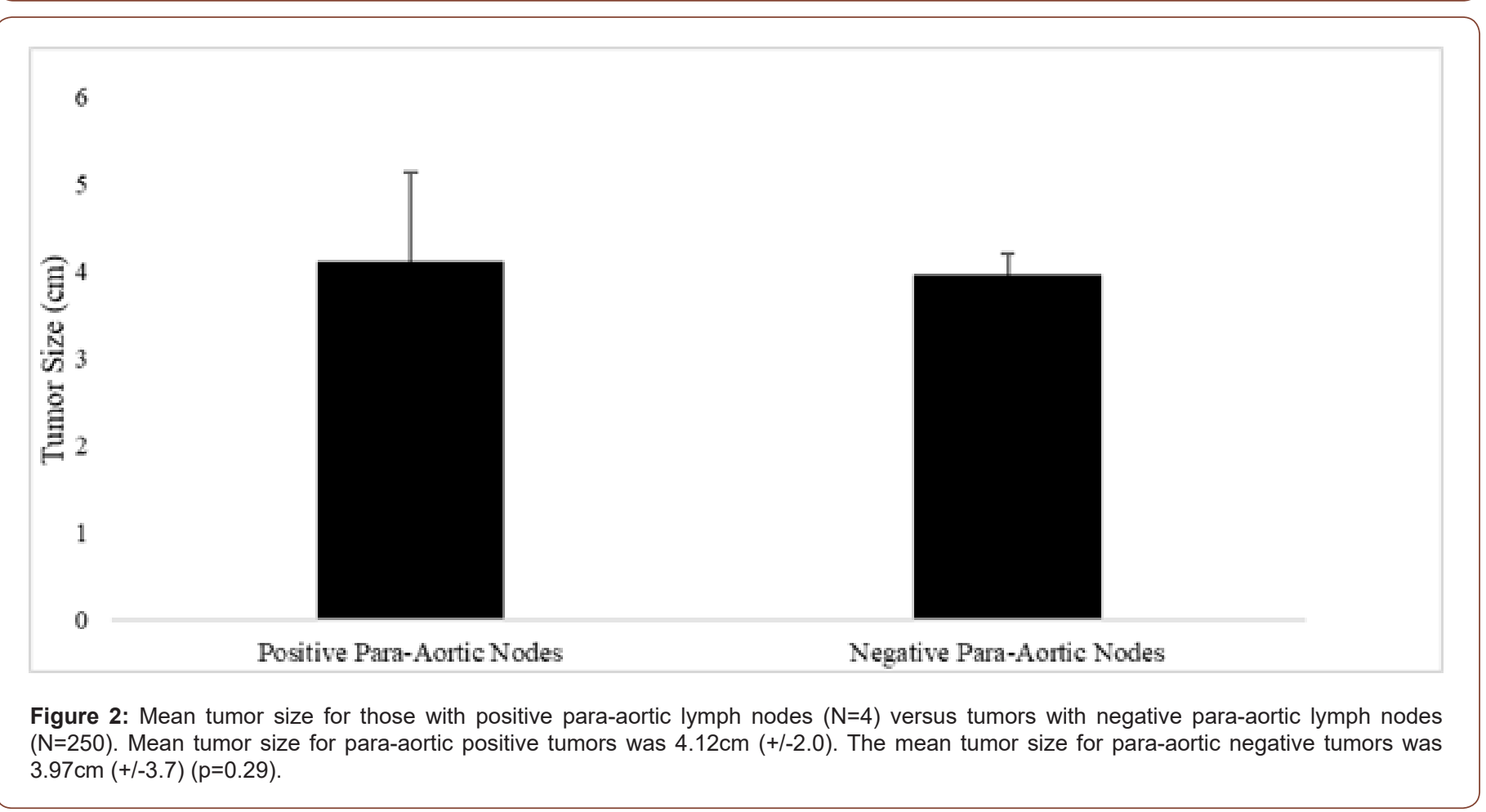




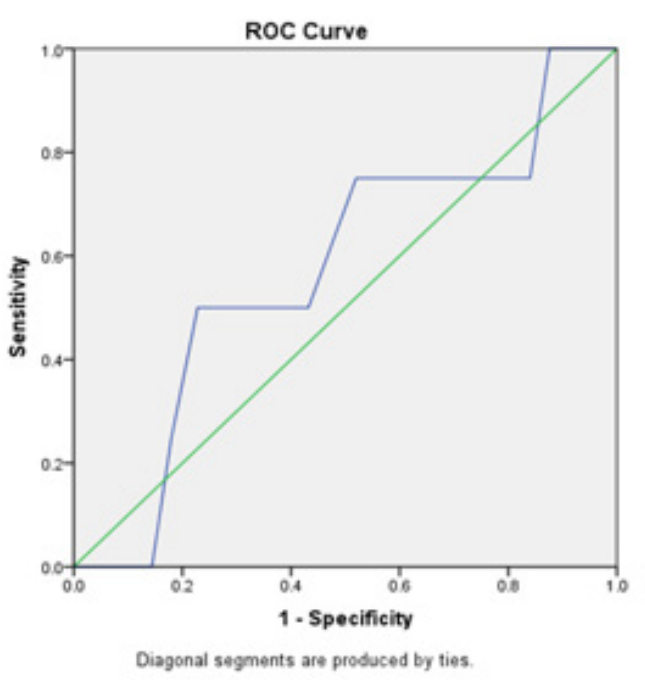

Figure 3: Receiver operating characteristic (ROC) curve for tumor size and positive para-aortic lymph nodes. Tumors measuring $5 \mathrm{~cm}$ or more were found to have the following associations with positive PALN involvement: a positive predictive value (PPV) of $2.94 \%$, a negative predictive value (NPV) of $98.92 \%$, sensitivity of $50.00 \%$ and specificity of $73.60 \%$ Area under the curve (AUC) 0.575 .

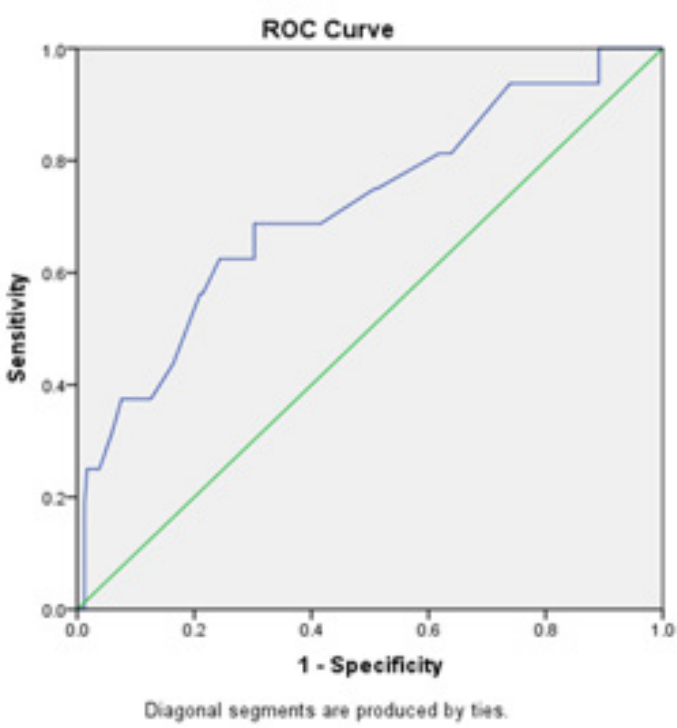

Figure 4: ROC Curve for tumor size and positive pelvic lymph nodes. Tumors of $5 \mathrm{~cm}$ or more were found to have the following associations with positive PLN involvement: PPV of $14.71 \%$, NPV of $96.77 \%$, sensitivity of $62.50 \%$ and specificity of $75.63 \%$. AUC 0.718 .

\section{Tumor location}

Tumor location, which was defined as "lower uterine segment" or "fundus", was compared to pelvic and para-aortic lymph node involvement. Three of the four patients $(75 \%)$ who had positive PALN identified, had a tumor located in the fundal region. Of the 254 patients without PALN involvement, 179 (70.50\%) also had tumors located in the fundal region ( $\mathrm{p}=1.00$, Figure 5). Fundal tumors had a PPV of $1.66 \%$, NPV of $98.70 \%$, SN of $75.00 \%$ and SP of $29.92 \%$ for PALN involvement. When comparing PLN metastasis and tumor location; 7 out of the total 16 positive pelvic node samples had a tumor located in the uterine fundus (43.75\%). Out of the 242 negative pelvic node specimens, 174 samples (71.90\%) were also fundal tumors $(p=0.02)$. Fundal tumors had a PPV of $3.87 \%$, NPV of $88.31 \%$, sensitivity of $43.75 \%$ and specificity of $28.10 \%$ for PLN involvement.

\section{Myometrial Invasion}

Myometrial invasion was not statistically significant between positive and negative PALN samples. Mean percentage of myometrial invasion for tumors with positive para-aortic lymph nodes $(\mathrm{N}=4)$ versus tumors with negative para-aortic lymph nodes $(\mathrm{N}=255)$ was compared. Mean myometrial invasion for para-aortic positive tumors was $50.25 \%(+/-18.8)$. The mean myometrial invasion for para-aortic negative tumors was 31.3\% (+/29.59) $(\mathrm{p}=0.14$, Figure 6). Results comparing pelvic and para-aortic lymph node involvement were also dichotomized by the median of 
myometrial invasion percentage of $28 \%$. All four patients identified with positive PALN involvement had greater than $28 \%$ myometrial invasion. Of the remaining 255 patients, 125 samples also had $>28 \%$ myometrial invasion, producing a PPV of $3.10 \%$, NPV of $100.0 \%$, sensitivity of $100.0 \%$ and specificity of $50.98 \%$ (Figure $7)$. In the positive PLN samples $(\mathrm{N}=16)$, myometrial invasion had a reported median of $71 \%$ compared to a median of $26 \%$ invasion in the negative group $(\mathrm{N}=243)(\mathrm{p}<0.001)$. Patients identified with PLN metastasis and $>28 \%$ myometrial invasion were calculated to have a PPV of $10.85 \%$, NPV of $98.46 \%$, sensitivity of $87.50 \%$, and specificity of $52.67 \%$ (Figure 8 ).

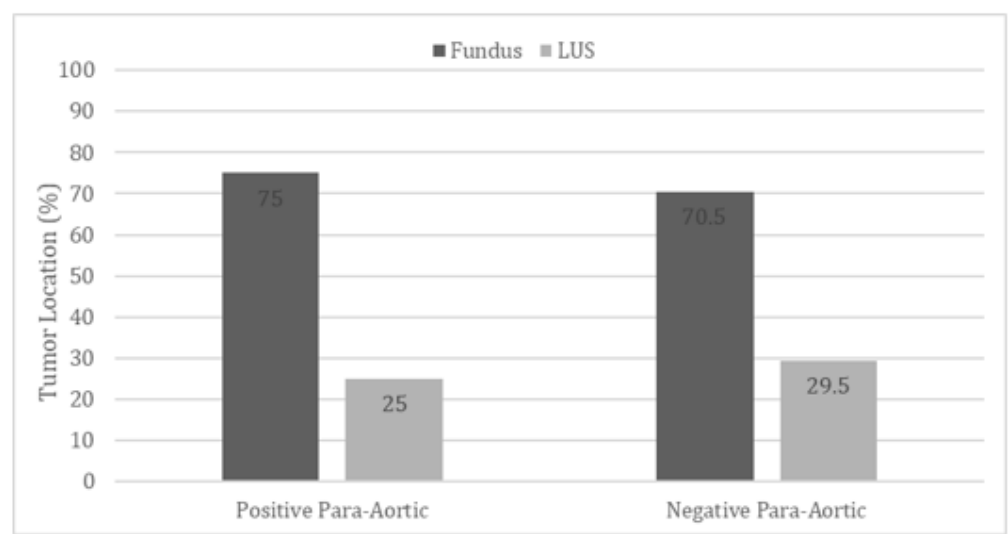

Figure 5: Percentage of tumors located in the fundus and lower uterine segment (LUS) for positive para-aortic lymph nodes ( $N=4)$ versus negative para-aortic lymph nodes ( $\mathrm{N}=255)$. Tumors with positive para-aortic lymph nodes occurred in the fundus in $75 \%$ of cases and in the LUS (lower uterine segment) in $25 \%$ of cases. Tumors with negative para-aortic lymph nodes were found in the fundus in $70.5 \%$ of cases and in the LUS in $29.5 \%$ of cases $(p=1.0)$.

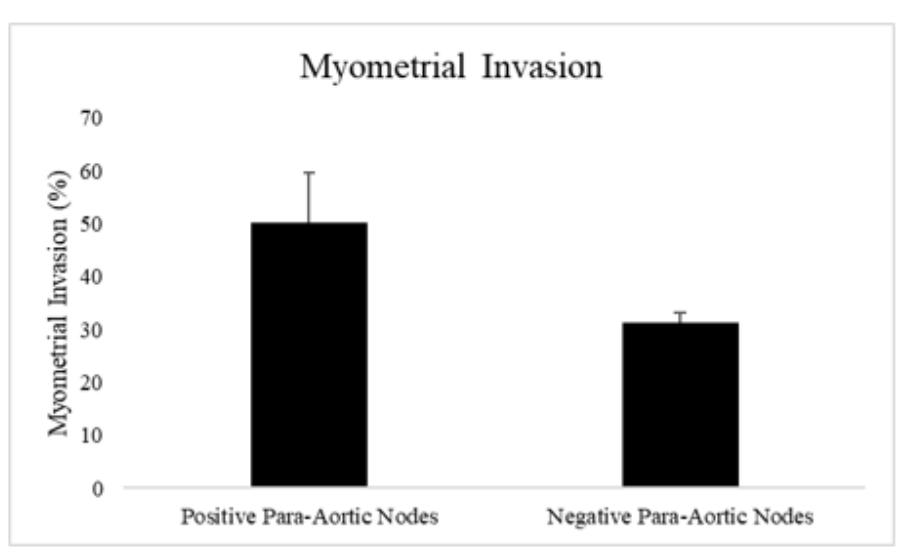

Figure 6: Mean percentage of myometrial invasion for tumors with positive para-aortic lymph nodes $(\mathrm{N}=4)$ versus tumors with negative paraaortic lymph nodes $(\mathrm{N}=255)$. Mean myometrial invasion for para-aortic positive tumors was $50.25 \%(+/-18.8)$. The mean myometrial invasion for para-aortic negative tumors was $31.3 \%(+/-29.59)(p=0.14)$.

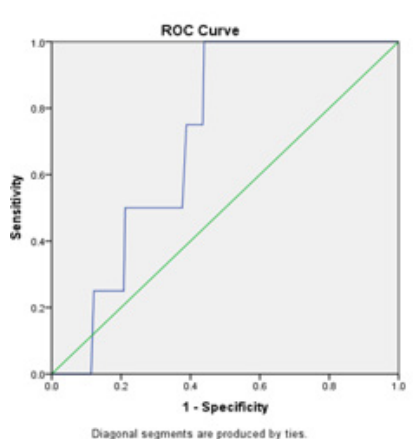

Figure 7: ROC Curve. Myometrial invasion and positive para-aortic lymph nodes. All four patients identified with positive PALN involvement had greater than $28 \%$ myometrial invasion. Of the remaining 255 patients, 125 samples also had $>28 \%$ myometrial invasion, producing a PPV of $3.10 \%$, NPV of $100.0 \%$, sensitivity of $100.0 \%$ and specificity of $50.98 \%$. AUC 0.713 . 


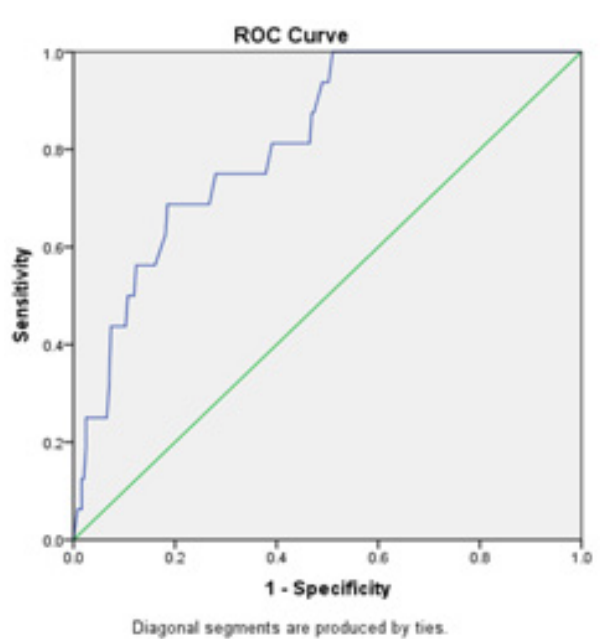

Figure 8: ROC Curve. Myometrial invasion and positive pelvic lymph nodes. PLN metastasis and $>28 \%$ myometrial invasion was calculated to have a PPV of $10.85 \%$, NPV of $98.46 \%$, sensitivity of $87.50 \%$, and specificity of $52.67 \%$. AUC $=0.814$.

\section{LVSI}

Secondary findings comparing lymph node metastasis and LVSI were also identified. LVSI was found to significantly correlate with positive lymph node samples in both para-aortic and pelvic specimens. Of the four patients identified with positive PALN, $100 \%$ had positive LVSI compared to positive LVSI in $22.31 \%$ of the negative para-aortic specimens ( $p=0.003$, Figure 9). LVSI was also found to independently correlate to pelvic lymph node involvement. Of the 16 samples identified with positive pelvic nodes, $81.25 \%$ ( $\mathrm{n}=13$ ) had positive LVSI, compared to only $19.67 \%$ $(\mathrm{n}=47)$ of negative pelvic lymph node specimens with positive LVSI $(\mathrm{p}<0.001)$.

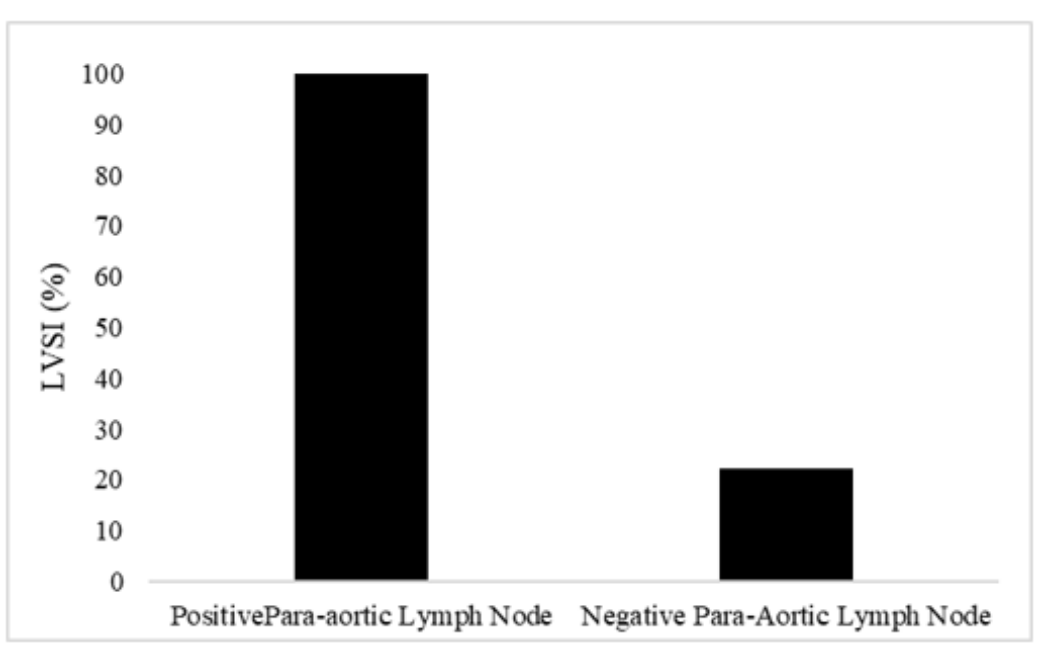

Figure 8: Percentage of tumors with positive lymphovascular space invasion (LVSI) for positive para-aortic lymph nodes ( $N=4)$ versus negative para-aortic lymph nodes $(\mathrm{N}=251)$. Of the tumors with positive para-aortic lymph nodes, $100 \%$ had $\mathrm{LVSI}$. The tumors with negative para-aortic lymph nodes had LVSI at a rate of $22.31 \%(p=0.003)$.

\section{Discussion}

The Mayo criteria, established in 2004, is a widely recognized algorithm for lymph node dissection in the management of endometrial cancer5. The criteria states that the following tumor characteristics do not require lymph node dissection: histologic FIGO (International Federation of Gynecology and Obstetrics) grades 1 or 2 , with myometrial invasion less than or equal to $50 \%$, and primary tumor diameter less than or equal to $2 \mathrm{~cm}$. Additionally, patients who do not meet this criteria, with tumor size of greater than $2 \mathrm{~cm}$ and myometrial invasion greater than $50 \%$ or have non-endometrioid histology, should undergo both pelvic and para-aortic lymph node biopsies [5]. These guidelines have been supported by additional literature stating that patients with low risk endometrial cancer have a low rate of lymph node metastasis [11]. Despite recommendations, para-aortic lymph node dissection is still not performed as routinely as pelvic nodal dissection even in appropriate cases, potentially due to the technical complexity and associated risk profile. Para-aortic lymph node dissection is surgically more challenging and carries a higher morbidity [12], 
so it is essential to determine if any specific predictive factors can be identified to assist surgeons in this complex operative decision making.

Based on our study results, when looking at independent predictive factors of PALN involvement in low grade endometrial cancer, LVSI was the only variable found to be statistically significant. Tumor size, location, and myometrial invasion were not found to be significant predictive factors, although some findings such as the negative predictive values, sensitivity, and specificity produced could still offer support of clinical guidance. LVSI has been previously studied as an independent factor for PLN involvement $[3,13]$ and has been found to be highly predictive of nodal disease in some low-grade endometrial cancers. In a previous study by Kumar et al. looking specifically at para-aortic lymphadenectomy, it was found that the rate of PALN metastasis in patients with endometrioid type endometrial cancer without LVSI was only $0.80 \%$ [14]. Our study demonstrates that of the samples with positive PALN involvement, $100.00 \%$ had LVSI, compared to only $22.31 \%$ of the negative PALN samples $(\mathrm{p}=0.003)$. These findings further support the predictive value of LVSI on PALN involvement in low grade endometrial cancers.

Although myometrial invasion, tumor size, and tumor location did not produce significant outcomes for PALN in our study, secondary results generated are still warranted to discuss. Myometrial invasion showed significance when looking at the relation with pelvic lymph node involvement, with a median of $71 \%$ invasion. A median of $48 \%$ invasion was seen with paraaortic lymph nodes, although this finding was not significant. These results further support the previously described Mayo criteria5. Our findings also showed tumors measuring greater than $5 \mathrm{~cm}$ had a negative predictive value of $98.92 \%$ for PALN involvement and a negative predictive value of $96.77 \%$ for PLN metastasis. In regard to tumor location, although the sample size was small, 3 out of the 4-positive para-aortic lymph node samples were found to be located in the uterine fundus, producing a negative predictive value of $98.7 \%$ for PALN involvement. The present findings may suggest that tumors smaller than $5 \mathrm{~cm}$ and tumors located outside of the fundal region are less likely to have PALN metastases.

Further research should be conducted to evaluate these predictive factors of lymph node involvement in larger populations and also with different histologic type endometrial cancers. Overall survival rate has been shown to be higher in patients with complete lymphadenopathy in endometrial cancer [15], but again this is not without the risk of extensive dissection. This debated topic should be continued to be studied as it is essential to improve the recommendations and indications for lymph node dissection.

Our study is not without limitations. First, the sample size of 20 patients with positive nodal samples is extremely low. While we attribute this to our strict inclusion criteria, our actual sample size was much lower than anticipated. Given the low rates of PALN involvement in low grade endometrial cancer, this study may have benefited from a larger sample size or a case-control study design. Second, our study included patients from several surgeons. This may have introduced bias into the results, as diversity in surgical techniques may have contributed to a discrepancy between the number of nodes collected during each case. Finally, given the retrospective nature of the study, a portion of the patient charts and pathology reports did not include all of the information desired for the study.

\section{Conclusion}

Lymphovascular space invasion may be an independent predictor of both para-aortic and pelvic lymph node involvement in low grade endometrial tumors. It remains unclear whether tumor size, location, and myometrial invasion can be used to predict para-aortic nodal involvement in these cases. Additional research is needed before these factors should be used to dictate treatment algorithms or surgical decisions.

\section{Acknowledgement}

Adeima Ibanga BS, Simran Kripalani BS, Anjali Sivendra MS, BS

\section{Conflict of Interest}

Authors declare no conflict of interest.

\section{References}

1. Worldwide cancer data (2018) World Cancer Research Fund.

2. (2020) Key Statistics for Endometrial Cancer.

3. Zhang C, Wang C, Feng W (2012) Clinicopathological risk factors for pelvic lymph node metastasis in clinical early-stage endometrioid endometrial adenocarcinoma. Int J Gynecol Cancer 22(8): 1373-1377.

4. Chi DS, Barakat RR, Palayekar MJ, Levine DA, Sonoda Y, et al. (2008) The incidence of pelvic lymph node metastasis by FIGO staging for patients with adequately surgically staged endometrial adenocarcinoma of endometrioid histology. Int J Gynecol Cancer 18(2): 269-273.

5. Mariani A, Dowdy SC, Cliby WA, Gostout BS, Jones MB, et al. (2008) Prospective assessment of lymphatic dissemination in endometrial cancer: A paradigm shift in surgical staging. Gynecolo Oncol 109(1): $11-18$.

6. ASTEC study group, Kitchener H, Swart AMC, Qian Q, Amos C, Parmar MKB (2009) Efficacy of systematic pelvic lymphadenectomy in endometrial cancer (MRC ASTEC trial): A randomised study. Lancet 373(9658): 125-136.

7. Akbayir 0, Corbacioglu A, Goksedef BPC, Numanoglu C, Akca A, et al. (2012) The novel criteria for predicting pelvic lymph node metastasis in endometrioid adenocarcinoma of endometrium. Gynecol Oncol 125(2): 400-403.

8. Morrow CP, Bundy BN, Kurman RJ, Creasman WT, Heller P, et al. (1991) Relationship between surgical-pathological risk factors and outcome in clinical stage I and II carcinoma of the endometrium: A Gynecologic Oncology Group study. Gynecol Oncol 40(1): 55-65.

9. Creasman WT, Morrow CP, Bundy BN, Homesley HD, Graham JE, et al. (1987) Surgical pathologic spread patterns of endometrial cancer. A Gynecologic Oncology Group Study. Cancer 60(8 Suppl): 2035-2041.

10. Hirahatake K, Hareyama H, Sakuragi N, Nishiya M, Makinoda S, et al. (1997) A clinical and pathologic study on para-aortic lymph node metastasis in endometrial carcinoma. J Surg Oncol 65(2)82-87. 
11. Korkmaz V, Meydanli MM, Yalçın I, Sarı ME, Sahin H, et al. (2017) Comparison of three different risk-stratification models for predicting lymph node involvement in endometrioid endometrial cancer clinically confined to the uterus. J Gynecol Oncol 28(6): e78.

12. Volpi L, Sozzi G, Capozzi VA, Ricco’ M, Merisio C, et al. (2019) Long term complications following pelvic and para-aortic lymphadenectomy for endometrial cancer, incidence and potential risk factors: A single institution experience. Int J Gynecol Cancer 29(2): 312-319.

13. Guntupalli SR, Zighelboim I, Kizer NT, Zhang Q Powell MA, et al. (2012) Lymphovascular space invasion is an independent risk factor for nodal disease and poor outcomes in endometrioid endometrial cancer. Gynecol Oncol 124(1): 31-35.

14. Kumar S, Mariani A, Bakkum-Gamez JN, Weaver AL, McGree ME, et al. (2013) Risk factors that mitigate the role of paraaortic lymphadenectomy in uterine endometrioid cancer. Gynecol Oncol 130(3): 441-445.

15. Todo Y, Kato H, Kaneuchi M, Watari H, Takeda M, et al. (2010) Survival effect of para-aortic lymphadenectomy in endometrial cancer (SEPAL study): A retrospective cohort analysis. Lancet 375(9721): 1165-1172. 